\title{
Dental malocclusions are not just about small and weak bones: assessing the morphology of the mandible with cross-section analysis and geometric morphometrics
}

\author{
Viviana Toro-Ibacache ${ }^{1,2}$ (D) Francisco Ugarte ${ }^{3} \cdot$ Cristina Morales $^{3} \cdot$ Andrea Eyquem $^{4,5} \cdot$ José Aguilera $^{6}$. \\ Williams Astudillo ${ }^{6}$
}

Received: 10 July 2018 / Accepted: 4 December 2018 / Published online: 3 January 2019

(C) The Author(s) 2019

\begin{abstract}
Objectives Dental malocclusions in modern populations would be the result of small and weak jaws developing under low masticatory loads. We assess the validity of this by characterising the external and internal morphology of mandibles affected by class II and III malocclusions and comparing them with those from individuals with different masticatory load patterns.

Materials and methods CTs from up to 118 individuals exerting intensive, medium and low masticatory loads with harmonic occlusion, and from class II and III individuals, were used to compare their external shape using geometric morphometrics, as well as their internal amount and distribution of cortical bone.

Results The low-load groups (harmonic, class II and III occlusion) are externally more gracile than the intense and medium load groups. But more relevant in shape variation is a marked allometric pattern, which differentiates class II (small) and III (large) mandibles. Despite gracility, the relative amount of cortical bone in the low-load groups is larger than in the remaining groups. Conclusions There is no evidence that the modern mandible, including class II and III individuals, is intrinsically small and weak. Instead, there is a rather large degree of morphological variation, which could be linked to a lack of constraints derived from low masticatory loads. Thus, the effect of other factors such as genetics, but also basal metabolism, should be looked in more depth. Clinical relevance Dental malocclusions are a common disorder whose aetiology has not been unravelled, and several to be considered in the prevention and therapy of malocclusion.
\end{abstract}

Keywords Mandible shape $\cdot$ Cortical bone $\cdot$ Malocclusions $\cdot$ Diet consistency $\cdot$ Masticatory load

\section{Introduction}

Dental malocclusions are considered a "disease of civilization" [1]. They are common in current western populations, and markedly less frequent in archaic human populations [2] whose diet was composed mainly of more mechanically challenging items like meat (non-highly processed), seeds and abrasive elements in them [3, 4]. They are also less frequent in modern

Electronic supplementary material The online version of this article (https://doi.org/10.1007/s00784-018-2766-6) contains supplementary material, which is available to authorized users.

Viviana Toro-Ibacache

mtoroibacache@odontologia.uchile.cl; viviana_toro@eva.mpg.de

1 Centro de Análisis Cuantitativo en Antropología Dental and Instituto de Investigación en Ciencias Odontológicas, Facultad de Odontología, Universidad de Chile, Olivos 943, Independencia, Santiago, Chile

2 Department of Human Evolution, Mac Planck Institute for Evolutionary Anthropology, Deutscher Platz 6, 04103 Leipzig, Germany
Centro de Análisis Cuantitativo en Antropología Dental, Facultad de Odontología, Universidad de Chile, Santiago, Chile

4 Departamento de Antropología, Facultad de Ciencias Sociales, Universidad de Chile, Santiago, Chile

5 Max Planck Weizmann Center for Integrative Archaeology and Anthropology, Max Planck Institute for Evolutionary Anthropology, Leipzig, Germany

6 Facultad de Medicina and Hospital Clínico, Universidad de Chile, Santiago, Chile 
populations with more ancestral lifestyles whose diet consists of less processed items, and where infections leading to premature tooth loss are less common $[1,5]$. Tooth loss, and particularly a reduction in masticatory load, has been linked to the transition between hunter-gatherer and agricultural (also urban and modern) subsistence economies, causing a reduction in jaw size [6-8]. Traditionally in dentistry and physical anthropology, small jaws have been related to alterations like tooth crowding and an altered maxillomandibular relationship [1, 9-11].

Experimentally, it has been observed that diminished masticatory forces are capable of altering adult cranial and mandibular shape. In mice, for example, a diet based on soft food results in a shorter narrow rostrum, a short coronoid process, a small articular surface of the condyle and labially inclined incisors [12]. Moreover, inducing masseter muscle paralysis in adult mice leads to an increased activity of the bone resorption marker RANKL, followed by a reduction of bone tissue per area and trabecular thickness of the condyle head [13]. Using medical data from modern populations, it has been observed that muscle force is only weakly related to cranial shape [14], but more so to the shape of the mandible [15].

The developmental origin of dental malocclusions, particularly class II and III maxillomandibular relationships, has been difficult to pinpoint. There would be a genetic predisposition, to which habits and muscle malfunctioning would significantly contribute $[16,17]$. Class II and III phenotypes are also very variable and thus difficult to relate to isolated factors. Class II individuals can vary in their vertical component as well as their occlusal relationship [16]. For example, Hong and Yi [18], using a modified Delaire's cephalometric analysis, were able to identify six types of class III morphologies that relate to differences in the intra-individual arrangement of other cranial structures. The effect of function on mandible shape deformities would explain the differences in size found by Park, Kim, Yu, Yi and Lee [19] in the form of the functional units (i.e. areas affected by the surrounding functional tissues) of the mandible among skeletal classes I, II and III. However, the same study does not find differences in the size of the whole mandibles, and in some cases, the affected mandibles show larger linear dimensions in the functional areas.

One of the features that are common in class II and III mandibles is the vertical elongation of the face, either more anteriorly, posteriorly or both [20, 21]. From a structural perspective, a gracile vertically elongated mandible, reduced in bone mass, is a weak mandible [22] due to its reduced capacity to withstand torsional forces that arise during mastication [23, 24]. However, mandibular robusticity, estimated from external linear measurements and measurements of cortical bone amount and inner distribution, is not always a trait present in individuals exerting large masticatory loads [25].

Are then class II and III mandibles small and weak due to their reduced masticatory load, which is a characteristic of modern populations? Due to the nature of linear measurements, of common use in dentistry, the three-dimensional geometry of class II and III mandibles is not completely known. We aim to perform a detailed characterisation of the shape, size (in relation to shape, i.e. allometry) and inner morphology of mandibles affected by class II and III malocclusions (in the broad sense). We combine 3D shape analysis techniques (geometric morphometrics) and traditional crosssection analyses of the internal amount and distribution of cortical bone. Using a novel approach in dentistry that combines knowledge from the dental and anthropological sciences, we use archaeological and current populations that differ in their subsistence strategy (and thus diet consistency) and maxillomandibular relationship to test the null hypothesis that there are no differences in mandibular shape, amount and distribution of cortical bone among groups. If indeed, class II and III mandibles are morphologically modern mandibles that are particularly affected by the masticatory loads derived from the modern soft diet, we expect that they represent more extreme versions of the modern individuals with a harmonic maxillomandibular relationship. That is, that they show more gracile features together with small size and less amount of cortical bone, more elliptically distributed within the mandible's cross sections.

\section{Materials and methods}

Shape and structural analyses were performed using the CT scans of individuals from archaeological and modern samples present in anthropological collections and clinical databases. All individuals were adults, as assessed by the eruption of the third molar and/or the ossification of the spheno-occipital synchondrosis in their corresponding crania. In the case of the archaeological sample, a different number/list of individuals was used for each analysis due to their different status of conservation (e.g. there were individuals with or premature unilateral tooth loss or museum individuals with one side destroyed, not suitable for shape analysis but that could be used in cross-sectional analysis). In total, 118 individuals underwent mandible shape analysis, while 118, 97 and 97 individuals were included in cross-sectional geometry analyses of the symphysis, left and right first molar areas respectively. They correspond to current and past populations of current Chilean and Argentinean territories (Table 1). In the case of individuals in the clinical databases, their CTs were obtained for medical reasons, unrelated to this study. The skulls of the remaining individuals are housed in different museums and universities in Chile, Argentina and Italy (as described in Table 1), and were selected according to their conservation status and their dental status (with at least one molar in occlusion to ensure the presence of masticatory loads). The classification of the sample according to their masticatory load was made following previous anthropological studies $[4,6-8,25,32]$. These rely on the changes in 
Table 1 Sample description

\begin{tabular}{|c|c|c|c|}
\hline $\begin{array}{l}\text { Group } \\
\text { (load) }\end{array}$ & Institution & Geographical origin & Dating* \\
\hline \multirow[t]{3}{*}{ Intense } & FACSO (Chonos Collection) & South of Chile & $1050-650 \mathrm{BP}[26]$ \\
\hline & MLP (Chubut Collection) & $\begin{array}{l}\text { North East of the Argentinean } \\
\text { Patagonia }\end{array}$ & $1500-500$ ВР [27] \\
\hline & $\begin{array}{l}\text { MLP and MHNF (Fueguinos } \\
\text { Collection) }\end{array}$ & Tierra del Fuego, Argentina & $450-50$ ВР $[3,28]$ \\
\hline \multirow[t]{4}{*}{ Medium } & FACSO (Tarapacá 8 Collection) & North of Chile & $3060-1410$ ВР [29] \\
\hline & FACSO (Pica 8 Collection) & North of Chile & $1286-552$ cal BP [30] \\
\hline & MLP (Pampa Grande Collection) & North West of Argentina & $400-650 \mathrm{AD}[31]$ \\
\hline & MLP (Mapuche Collection) & $\begin{array}{l}\text { North of the Argentinean } \\
\text { Patagonia }\end{array}$ & s. XVII-XVIII [58] \\
\hline \multirow[t]{2}{*}{ Low } & FACSO (Sub Actal Collection) & Central Chile & $\begin{array}{l}\text { Sub Actual, 1950-1970 } \\
\text { [59] }\end{array}$ \\
\hline & HCUCh Clinic & Central Chile & Current era \\
\hline $\begin{array}{l}\text { Low-class } \\
\quad \text { II }\end{array}$ & ICOR Clinic & Central Chile & Current era \\
\hline $\begin{array}{l}\text { Low-class } \\
\text { III }\end{array}$ & ICOR Clinic & Central Chile & Current era \\
\hline
\end{tabular}

*As mentioned in the citing literature

FACSO, Facultad de Ciencias Sociales, Universidad de Chile; MLP, Museo de La Plata, Argentina; MHNF, Museo di Storia Naturale, Università di Firenze, Italy; HCUCh, Hospital Clínico Universidad de Chile, Chile; ICOR, Instituto de Cirugía Ortodoncia Rehabilitación Oral y Máxilofacial, Chile

subsistence economy from ancient to current populations, and what food items were common in them. The most ancestral type of subsistence economy was based in hunting animals and gathering fruits and/or seeds. As populations became sedentary, agriculture and cooking technologies developed, and vegetables replaced animals as the main resource. Meat is mechanically more challenging to chew, and cooking changes the material properties of food in mouth from hard/tough to hard/soft $[33,34]$. Nowadays, food items are extensively processed both mechanically and chemically, resulting in a majority of soft foods. Tough (and less so, hard) food items require comparatively larger, more sustained occlusal forces and more masticatory cycles before swallowing $[35,36]$. In the present study, we have chosen three groups of individuals based on the intensity of masticatory loads: intense (composed by hunter-gatherers), medium (agriculturalists) and low (individuals from modern, urban populations). As study cases, we have included individuals with low masticatory loads and altered maxillomandibular relationships, namely skeletal classes II and III. A detailed description of the groups is given in Table 1.

The CTs where acquired in different places and under different acquisition protocols. Since the study of large-scale features is of interest (i.e. whole geometry rather than geometry of small areas) and preliminary exploratory analyses showed no differences among individuals of different origin within load groups, the possible differences in acquisition protocol were not considered as a problem. In the contemporary, sample sex was known, while in the archaeological sample, it was estimated using classic cranial and postcranial (when available) features described in Buikstra and Ubelaker [37] by physical anthropologists that worked in each subsample. In some cases, sex could not be determined and had to be assigned (see below).

Three-dimensional reconstructions of each individual were made via threshold-based and manual segmentation using the software Avizo v.9.1 (Science Visualization Group, Burlington, EE.UU.) and saved as polygon (.ply) files. Using these $3 \mathrm{D}$ reconstructions, two types of data were obtained: mandible shape and size data, and cortical bone amount and distribution.

Mandible shape and size Thirty-two landmarks were placed to represent the general geometry of the mandible as well as the mechanically relevant features (molar and incisor distance to the temporomandibular joint, height of the ramus, etc.). Landmark description is in Table 2 and their location is shown in Fig. 1. Landmark coordinates were obtained by a single observer (FU) using Avizo. Geometric morphometrics is a robust statistical technique for the study of shape changes in relation to underlying variables $[38,39]$, and has been increasingly used in the last two decades in dental sciences (see examples in $[11,21,40,41])$. In geometric morphometrics, the landmark sets are used to obtain shape variables (i.e. the geometric properties of an object that are invariant to scale, rotation and translation; [38]). These shape variables are in our study represented by Procrustes coordinates, which are the landmarks after being corrected for scaling, rotation and 
Table 2 Definition of landmarks depicted in Fig. 2a and b

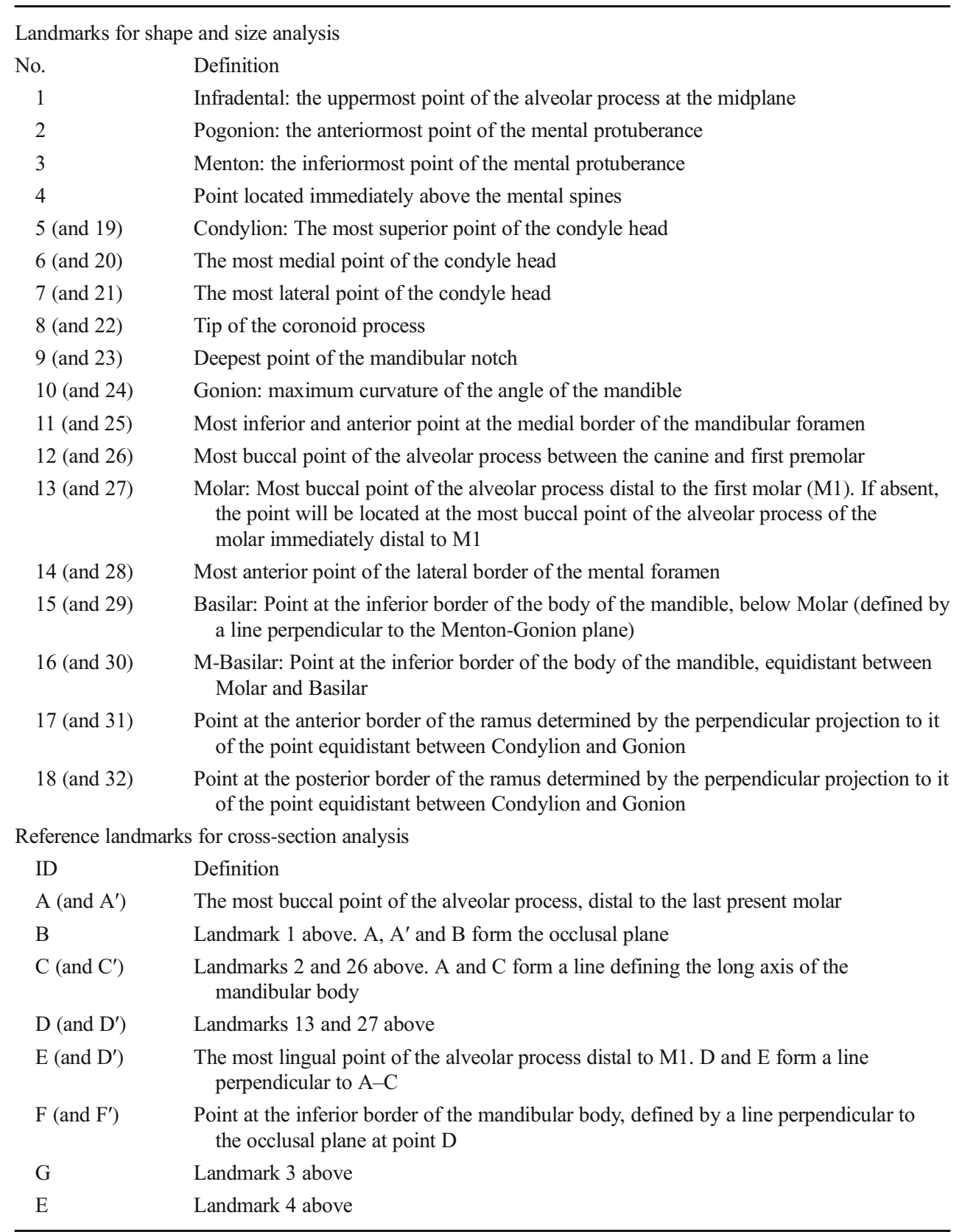

In parenthesis, numbers corresponding in the right side of the mandible translation. These shape variables are then compared among groups using multivariate statistics. During geometric morphometric analyses, the geometric size (named centroid size) of each configuration can be obtained and analysed as an independent variable.

Some preliminary analyses had to be performed. There were few individuals with missing landmarks due to bone damage. In these cases, the coordinates of these landmarks were estimated using the estimate.missing function of the Geomorph package [42] in R (www.r-project.org).

Sexual dimorphism is a factor that can modulate the relationship of shape with other variables. We ran procedures to eliminate it, based on the fact that the extent of its effect cannot be completely known. For example, sexual dimorphism is not a dominant characteristic in modern human populations, and it can reach very subtle levels in the mandible [43]. It has also been acknowledged that shape variation very unlikely reflects sexual dimorphism alone [44]. Moreover, the appearance of dimorphic traits can be altered by systemic factors such as malnutrition [45], whose presence cannot be discarded in observational studies of past populations. In order to run the statistical procedures to eliminate the effect of sexual dimorphism in our sample, we first had to assign sex to those individuals that remained undetermined after using the standard 
a

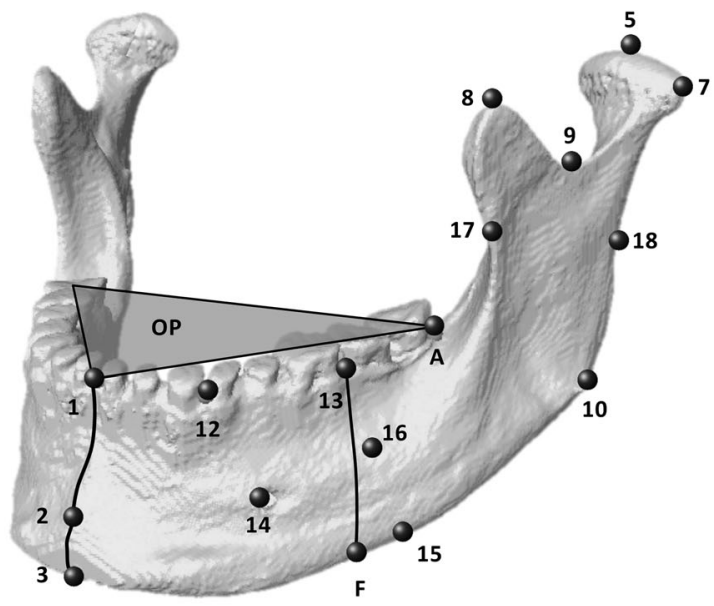

b

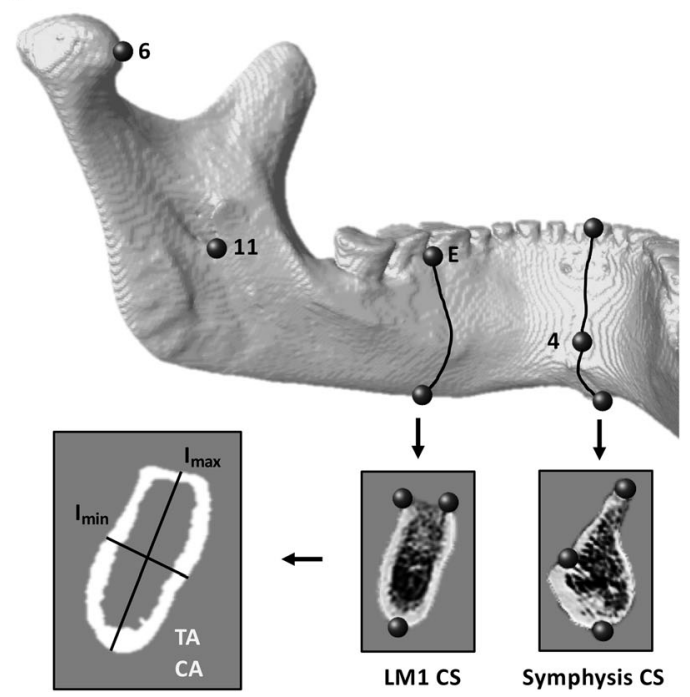

Fig. 1 The process of obtaining shape and cross-sectional geometry data. $\mathbf{a}$ and $\mathbf{b}$ show, in different views the landmarks defining mandible shape, the location of the occlusal plane (OP) and the planes for cross-sections (solid lines on the mandible). The landmarks of the right side of the mandible are not shown. A detailed definition of all the landmarks is given in Table 2. b Shows the images of cross-sections (CSs) obtained at the left molar (LM1) and symphysis area in order to measure the relative amount (TA-CA) and distribution $\left(\mathrm{I}_{\max }-\mathrm{I}_{\min }\right)$ of cortical bone anthropological procedure for it. For this, we used a discriminant function analysis in PAST [46], which assigned sex based on the shape of the most dimorphic individuals within load groups. Subsequently, the presence of sexual dimorphism as a confounding variable was confirmed performing a nonparametric, permutation-based multivariate analysis of variance (PERMANOVA; [47]) in PAST $(F=4.13 ; p<0.01)$. Following this, we used regression analysis in order to "remove" the weigh of those traits that could reflect more the effect of sex than of other factors. For this, shape variables were first corrected using a regression against sex as a dummy variable, where female $=-1$ and male $=1$ [48]. The residuals of the regression became then the new shape variables, free of the effect of sex, and they were subsequently subject to exploratory and confirmatory statistical analyses to test the hypothesis of this study.

To explore the general shape variation of the sample, principal component analyses (PCA) were performed in MorphoJ [49]. In order to reduce the number of dependent variables and to increase the statistical power of confirmatory (and also preliminary) tests, only the principal component scores explaining up to $75 \%$ of the explained variance were used as dependent variables, in PERMANOVAs with 9999 rounds of permutation. Pairwise tests with load groups as the independent variable followed PERMANOVA in PAST (see example in [12]). Allometric patterns of shape changes (i.e. shape changes related to centroid size changes), more than absolute size, are of importance to clarify the relevance of mandible size in dental malocclusions. Allometric shape changes were explored using multivariate regression of sex-corrected shape variables against centroid size in MorphoJ.
The total $n$ in this analysis was 118 . After sex assignation, 35 correspond to intensive load ( 10 women and 25 men), 38 to medium load (26 women and 12 men), 24 to low load (11 women and $13 \mathrm{men}$ ), 13 to low-class II load (10 women and 3 men) and 8 to low-class III load ( 2 women and 6 men).

Internal amount and distribution of cortical bone We designed a protocol based on the published literature $[25,50$, 51] in order to obtain $\mathrm{CT}$ images of with the minimal crosssection area at the mandibular symphysis, as well as immediately distal to the first molar of both sides. The procedure was performed by a single observer (CM). First, 13 landmarks were placed to identify key reference structures defining the occlusal plane and the corpus long axis (perpendicular to which cross-section planes are defined). These 13 landmarks are defined in Table 2 and shown in Fig. 1a. Once the crosssection planes are defined, a re-slicing of the $\mathrm{CT}$ volume data is performed, and the images obtained (Fig. 1b) are exported as tagged image file (.tiff). Landmark placing and crosssectional image generation were made in Avizo. The exported .tiff images were imported in MomentMacro (www. hopkinsmedicine.org/fae/mmacro.html) for ImageJ [52], and total bone area (TA), cortical bone area $(\mathrm{CA})$, minimum $\left(\mathrm{I}_{\min }\right)$ and maximum $\left(\mathrm{I}_{\max }\right)$ second moments of area were calculated (Fig. 1b). The relative amount of CA with respect to TA is an overall measure of rigidity (more cortical bone, more rigidity); $I_{\min }$ with respect to $I_{\max }$ represent cortical bone distribution (more circular distribution offers a better resistance to torsion and an elliptical distribution, to parasagittal bending) [25] These data are normally treated as ratios, which pose several problems for statistical analyses [53]. We decided then to use 
indexes representing the differences between the related values [54]: the difference between TA and CA, and between $I_{\max }$ and $I_{\min }$. In these cases, larger values of the former indicate low rigidity, and larger values of the latter, a more elliptic distribution of bone.

Since mandible shape presents sexual dimorphism, TA-CA and $I_{\max }-I_{\min }$ values were also corrected for sex using a linear regression of the values against sex as a dummy variable as in shape analysis, using PAST.

Sex-corrected TA-CA and $I_{\max }-I_{\min }$ values underwent Kruskal-Wallis test and pairwise Mann-Whitney comparison. Non-parametric tests were used as in all cases, as sexcorrected $\mathrm{I}_{\max }-\mathrm{I}_{\min }$ values were not normally distributed (Shapiro Wilk's $p<0.01$ ).

One hundred eighteen individuals were used in the symphysis analysis: 35 correspond to intensive load ( 8 women and 27 men), 39 to medium load ( 26 women and 13 men), 23 to low load (10 women and 13 men), 13 to low-class II load (10 women and 3 men) and 8 to low-class III load ( 2 women and 6 men). Ninety-seven individuals were used for the left molar area measurements: 34 of intensive load ( 7 women and 27 men), 23 of medium load (17 women and 6 men), 19 of low load ( 7 women and 12 men), 13 of low-class II load (10 women and 3 men) and 8 of low-class III load ( 2 women and 6 men). Ninety-seven individuals were also used to obtain right molar area data: 30 of intensive load ( 6 women and 24 men), 28 of medium load (women and 8 men), 18 of low load ( 8 women and 10 men), 13 of low-class II load (10 women and 3 men) and 8 of low-class III load ( 2 women and 6 men).

Measurement error In order to discard the effect of landmarking error on shape variation, a subsample of 20 individuals underwent repeated landmarking. The repeated landmark configurations were analysed using Procrustes analysis of variance (ANOVA) in MorphoJ with interindividual differences as factor and the differences within individuals as the error term.

The effect of measurement error on the raw values of TA, $\mathrm{CA}, \mathrm{I}_{\max }$ and $\mathrm{I}_{\min }$ values was assessed using a subsample of 20 individuals where the process of image extraction and measurement was performed twice. Data from both procedures were compared using the Friedman test for repeated measurements in PAST.

The method in this study was approved by the Ethical Committee of Facultad de Medicina, Universidad de Chile (CEISH, No. 203-2015).

\section{Results}

The effect of measurement error on results was neglectable on both mandible shape analyses, as interindividual variation was several times larger than intraindividual variation $(F=18.66$, $p<0.01$ ) and cross-section raw data (TA $\chi^{2}=0.01, p=1$; CA $\chi^{2}=0.83, p=0.37 ; \mathrm{I}_{\max } \chi^{2}=0.83, p=0.37 ; \mathrm{I}_{\min } \chi^{2}=0.01$, $p=1)$.

Shape analysis The first six principal components (PCs) explain up to $75 \%$ of the observed variance. Of these, only PC2 shows a slight separation between groups, specifically the archaeological from the modern sample. PC1, hierarchically the most relevant principal component describing overall shape variation, depicts features that are shared among groups, without separation between them except for low-class II and low-class III individuals (Fig. 2a). These features resemble allometric changes described below. In the second PC, both intensive and medium load groups are broadly overlapped, and they together overlap part of the low load group. Towards the modern group, the mandible tends to have more gracile features: more open mandibular angles, and slenderer rami and corpus at the level of posterior teeth. The PERMANOVA yielded statistically significant differences in shape among groups $(F=$ $10.49, p<0.01)$ and also between pairs of groups, except between the intensive and medium load groups after Bonferroni correction (Table 3).

Allometry has a significant effect on shape variation (variance predicted $3.96 \%, p<0.01$ ). All groups tend to cluster, but class II individuals are located more towards the smallest mandibles and class III, at the opposite end, together with several individuals from the intensive load group (Fig. 3). Larger mandibles show less bilaterally divergent rami, a more projected menton and overall, more mesially displaced corpus, while the ramus tends to displace distally, particularly the condyle (Fig. 2b).

Cross-section geometry The results of the statistical analyses of differences in TA-CA and $\mathrm{I}_{\max }-\mathrm{I}_{\min }$ (Tables 4, 5, 6, 7, 8 and 9) are based on the residuals of the raw values against sex. Since in this case residuals are not suitable for morphological and functional interpretations, we present their descriptive statistics in Tables S1 and S2 (Supplementary Information), and depict the raw values, pre-regression, as boxplots in Fig. 3.

TA-CA showed significant differences among groups at the symphysis $(H=19.82, p<0.01)$, left $(H=30.06, p<0.01)$ and right first molar $(H=32.12, p<0.01)$. After Bonferroni correction, differences remained significant between the extreme groups for the symphysis (intense vs. low and both altered occlusion groups) and between intense/medium load and low/low-class II individuals for the left and right first molar areas (Tables 4, 6 and 8 respectively). Overall, low, low-class II and low-class III groups tend to have a larger relative amount of cortical bone (that is, lower median values of TA-CA) when compared with the intensive and medium group (Fig. 3). Within groups, the symphysis has relatively more cortical bone than the molar areas (Fig. 3). 
Fig. 2 Shape and allometry analyses. a Principal component analysis, with first (PC1) vs. second (PC2) principal components shown. Insets depict shape changes associated to the extreme PC scores found, with the grey form as the mean, reference shape. b Allometry test: regression of shape components against centroid size. The insets represent shape changes, in different views, that occur with an increase of $150 \mathrm{~mm}$ in centroid size. Each individual is represented by a coloured circle. Load groups: red = intensive; green $=$ medium; blue $=$ low; grey $=$ low-class II; black = low-class III a

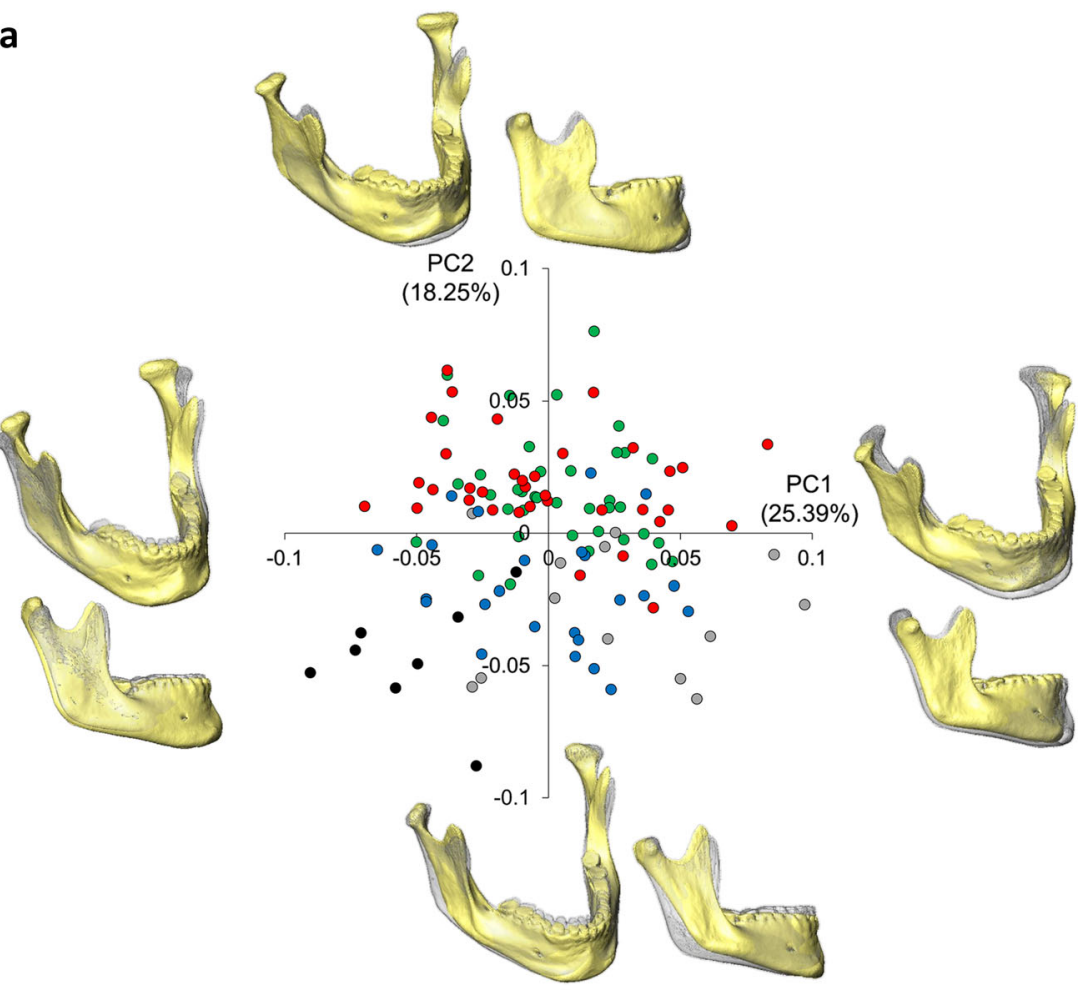

b

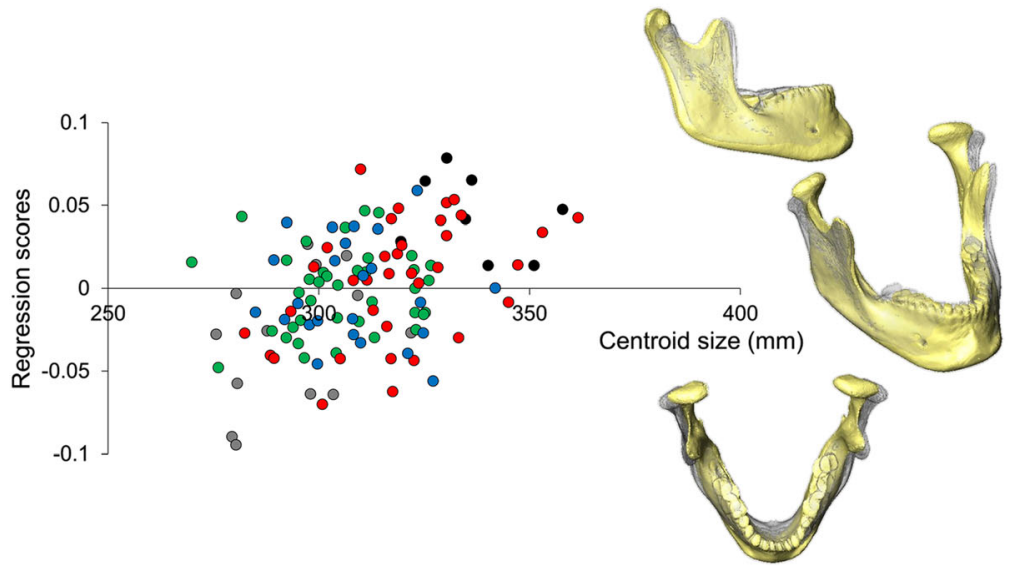

$I_{\max }-I_{\min }$ showed differences among groups in both molar areas ( $H=11$ and $p=0.03$ each side) but not in the symphysis $(H=5.59, p=0.23)$. However, no pairwise comparison yielded significant $p$ values after Bonferroni correction (Tables 5, 7 and 9). Beyond statistical significance and the large variation within groups, there are some subtle trends that
Table 3 Pairwise PERMANOVA of PC scores (PCs 1 to 6) between groups

\begin{tabular}{llllll}
\hline & Intense & Medium & Low & Low-class II & Low-class III \\
\hline Intense & & 2.48 & 10.77 & 14.52 & 16.61 \\
Medium & 0.040 & & 7.62 & 12.71 & 19.45 \\
Low & $1 \times 10^{-4}$ & $1 \times 10^{-4}$ & & 5.52 & 8.69 \\
Low-class II & $1 \times 10^{-4}$ & $1 \times 10^{-4}$ & $6 \times 10^{-4}$ & & 12.61 \\
Low-class III & $1 \times 10^{-4}$ & $1 \times 10^{-4}$ & $2 \times 10^{-4}$ & $1 \times 10^{-4}$ & \\
\hline
\end{tabular}

Upper diagonal shows $F$ values and lower diagonal, raw $p$ values. Significant $p$ values after Bonferroni correction are italicized 

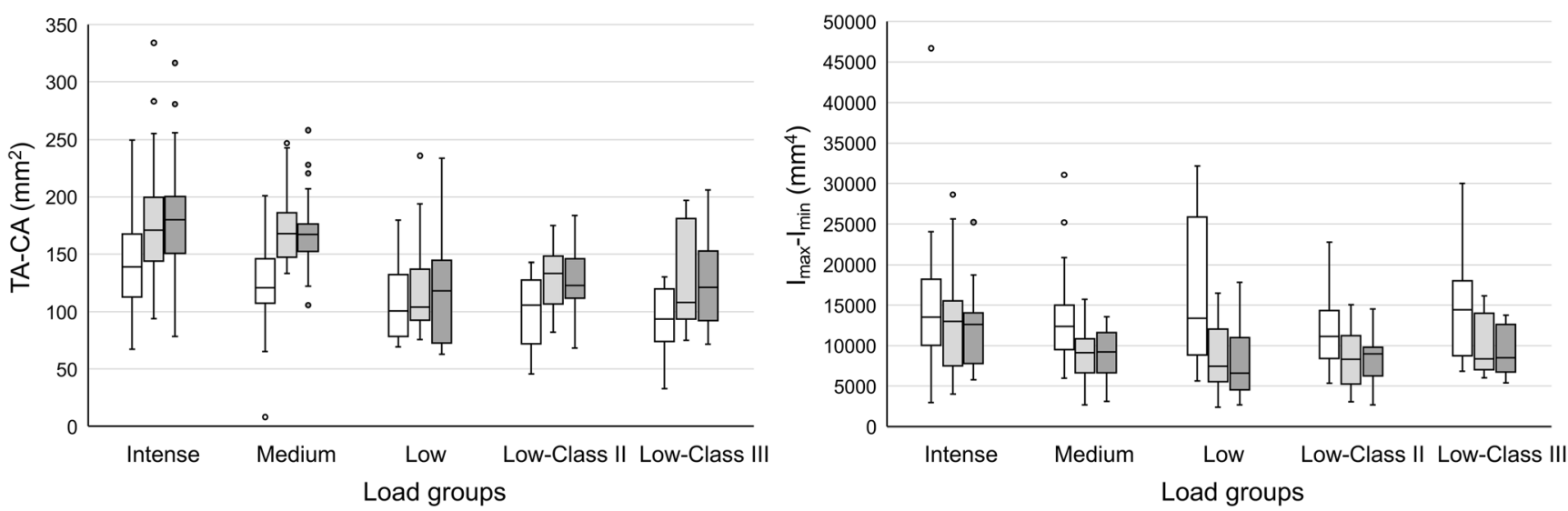

Fig. 3 Box plots of raw values of TA-CA and $I_{\max }-I_{\min }$. Medians, 25 and 75 percentiles, minimum and maximum values and outliers are shown. Each anatomical area is represented by a colour: white = symphysis; light grey = left molar; dark grey = right molar

can be observed. The intensive and medium load group tend to have a more circular distribution of cortical bone (i.e. lower median values of $I_{\max }-I_{\min }$ ) in the three measured areas. The three low load groups (i.e. low, low-class II and low-class II) on the other hand show more differences between sites, with the symphysis having a more markedly elliptical bone distribution (Fig. 3). Within groups, the molar areas have a more elliptical cortical bone distribution than the symphysis, without clear differences between sides (Fig. 3).

\section{Discussion}

The consideration of the modern (contemporary) human jaw as a small, weak structure when compared with its ancestors due to changes in diet has been long associated with the origin of dental malocclusions $[1,9,10]$. Using combined techniques and individuals from current and archaeological populations that differ in their subsistence economy and maxillomandibular relationships, the aim of this study was to perform a detailed characterisation of the shape, size and inner structure of the mandible in order to identify traits that could be linked to diet consistency and dental occlusion type.

Table 4 Mann-Whitney $U$ pairwise comparison of TA-CA residuals of the symphysis

\begin{tabular}{lcccll}
\hline & Intense & Medium & Low & Low-class II & Low-class III \\
\hline Intense & & 500 & 211 & 97 & 48 \\
Medium & 0.049 & & 304 & 159 & 182 \\
Low & 0.002 & 0.036 & & 143 & 79 \\
Low-class II & 0.003 & 0.047 & 0.843 & & 42 \\
Low-class III & 0.004 & 0.037 & 0.573 & 0.492 & \\
\hline
\end{tabular}

The upper diagonal shows the values of the $U$ statistic and the lower diagonal the raw $p$ values. $p$ values that reached statistical significance after Bonferroni correction are italicized
The null hypothesis that there are no differences in mandibular shape, size amount and distribution of cortical bone among groups could not be fully rejected. Although there were some differences among groups, these do not completely match the expectation that modern mandibles are intrinsically small and weak. On the contrary, there is a large variation within groups, leading to extensive overlaps. As expected though, class II mandibles are indeed smaller and show gracile features (they are located in the lower right quadrant in the PC plot in Fig. 2a and towards the left end in the allometry plot in Fig. 2b), while class III are larger and also gracile (Figs. 2 and $3)$. Gracility is a shared feature among the three low load groups, but the low load mandibles with harmonic occlusion are not small. In fact, they are slightly larger (i.e. they are located more to the right end of the $\mathrm{X}$ axis in Fig. 2b) than the medium load group. The larger mandibles belong to the intensive load group, but they have a wide range of sizes that includes those of the medium and low load groups.

Internal morphology shows less differences among groups. In terms of amount of cortical bone, however, the three low load groups have more than the intense and medium load groups (Fig. 3), which is against expectations: there is supposed to be a "need" of more cortical bone in mandibles that are subject to larger masticatory forces. The degree of

Table 5 Mann-Whitney $U$ pairwise comparison of $\mathrm{I}_{\max }-\mathrm{I}_{\min }$ residuals of the symphysis

\begin{tabular}{llllll}
\hline & Intense & Medium & Low & Low-class II & Low-class III \\
\hline Intense & & 494.5 & 335 & 191 & 120 \\
Medium & 0.042 & & 432 & 211 & 96 \\
Low & 0.287 & 0.816 & & 136 & 65 \\
Low-class II & 0.404 & 0.375 & 0.669 & & 38 \\
Low-class III & 0.543 & 0.092 & 0.232 & 0.328 & \\
\hline
\end{tabular}

The upper diagonal shows the values of the $U$ statistic and the lower diagonal the raw $p$ values. After Bonferroni correction, no $p$ values remained significant 
Table 6 Mann-Whitney $U$ pairwise comparison of TA-CA residuals of the left first molar area

\begin{tabular}{llllll}
\hline & Intense & Medium & Low & Low-class II & Low-class III \\
\hline Intense & & 348 & 95 & 76 & 72 \\
Medium & 0.489 & & 59 & 52 & 54 \\
Low & $2.45 \times 10^{-5}$ & $5.87 \times 10^{-5}$ & & 82 & 66 \\
Low-class II & 0.001 & 0.001 & 0.116 & & 47 \\
Low-class III & 0.042 & 0.090 & 0.614 & 0.745 & \\
\hline
\end{tabular}

The upper diagonal shows the values of the $U$ statistic and the lower diagonal the raw $p$ values. $p$ values that reached statistical significance after Bonferroni correction are italicized circularity of cortical bone distribution is very similar among groups.

Previous studies of external bone morphology, particularly from anthropological sciences, assign a rather high importance of masticatory load on bone morphology, with notorious differences between hunter-gatherer groups and agriculturalists [6-8]. Our results show far less differences among groups. Several factors could account for these differences in results, such as ancestry and sample composition (none of the other studies included modern individuals with altered occlusion). Also, the classification in groups according to masticatory loads presents limitations; the material properties of food items cannot be measured in extinct populations, nor strictly controlled in humans. Independently of the place or culture in which an individual grew, what he or she actually did in life cannot be known with certainty. However, it is worth to highlight that our study included individuals that had at least one molar in occlusion in order to ensure that masticatory loads were exerted in life. This is not trivial, since agriculturalists tend to show more infection-based tooth loss [1, 5], and tooth loss causes form changes in the mandible [55]. The dental status of the individuals included in the aforementioned anthropological studies is not explicit. Our results are more in agreement with Katz, Grote and Weaver [56], who found rather modest effects of subsistence economy on mandibular shape. They attribute more importance to other factors, linked to genetics, on shape changes over many generations,

Table 7 Mann-Whitney $U$ pairwise comparison of $I_{\max }-I_{\min }$ residuals of the left first molar area

\begin{tabular}{llllll}
\hline & Intense & Medium & Low & Low-class II & Low-class III \\
\hline Intense & & 233.5 & 190 & 121 & 100 \\
Medium & 0.011 & & 208 & 128 & 90 \\
Low & 0.014 & 0.801 & & 122 & 63 \\
Low-class II & 0.018 & 0.489 & 0.969 & & 41 \\
Low-class III & 0.256 & 0.946 & 0.507 & 0.447 & \\
\hline
\end{tabular}

The upper diagonal shows the values of the $U$ statistic and the lower diagonal, the raw $p$ values. After Bonferroni correction, no $p$ values remained significant particularly in closely related groups (from a similar ancestry) where genetic diversity and shape variation are linked.

Despite broad similarities of external morphology among groups with different masticatory load intensity, class II and III individuals show more marked differences with the rest of the groups, but less so to the low load group. Thus, the three modern samples together show a quite broad range of shapes, with class II and III mandibles in the extremes of the shape variation. This is linked to the allometric effect present in the sample, i.e. the shape of class II mandibles is dependent of their small size and the shape of class II mandibles, of their large size. Same as genetics, masticatory muscle force has shown to have an effect on mandibular shape in modern individuals [15]. But large shape variation leading to the extreme features shown by individuals with malocclusions could be linked to the effect of other factors, such as nutrition and basal metabolism in modern humans. This possibility has been largely overlooked and, unfortunately, it is far less straightforward to assess although there is some evidence that supports it. In pigs, it has been observed that the nutritional changes associated to weaning cause a decrease in skull growth rates, either when weaning occurs early or late in ontogeny [57]. This is surprising since weaning also changes the pattern of muscle activity, increasing jaw closing function (which should lead to an increased bone growth). Moreover, humans whose development and body size has been affected by diseases and malnutrition can recover later in development if properly nourished [26]. These two studies show that (1) within the life of an individual, the timing at which metabolic alterations occur is key [34], and (2) that nutrition (and hence metabolism) is an extremely relevant factor affecting bone morphology. This effect of metabolism could perhaps be comparable to that of mechanics on bone growth (at least in pigs and modern humans) or even larger, if mechanical constraints are reduced. At a larger, evolutionary time scale, the transition from hunting and gathering to agriculture in human populations has also, but less notoriously, been linked to smaller bones due to malnutrition [5] and hormonal changes related to a low-protein diet [27]. Our medium load group is comprised by agriculturalists, and indeed, they show relatively smaller sizes than the intensive load (comprised by huntergatherers) and the low and low-class III groups. These last 
Table 8 Mann-Whitney $U$ pairwise comparison of TA-CA residuals of the right first molar area

\begin{tabular}{llllll}
\hline & Intense & Medium & Low & Low-class II & Low-class III \\
\hline Intense & & 343 & 93 & 44 & 48 \\
Medium & 0.234 & & 91 & 52 & 41 \\
Low & $1.71 \times 10^{-4}$ & $3.03 \times 10^{-4}$ & & 97 & 60 \\
Low-class II & $6.90 \times 10^{-5}$ & $2.86 \times 10^{-4}$ & 0.435 & & 47 \\
Low-class III & 0.01 & 0.007 & 0.523 & 0.745 & \\
\hline
\end{tabular}

The upper diagonal shows the values of the $U$ statistic and the lower diagonal the raw $p$ values. $p$ values that reached statistical significance after Bonferroni correction are italicized two, in the case of our Chilean population, were likely exposed to a more balanced diet than agriculturalists. Class II individuals would be the exception to this. Thus, the link between a reduced masticatory loads and dental malocclusions in modern, urban populations is not as straightforward as suggested. Unlike other animals, humans are able to reach adult size without the need of exerting large masticatory loads. It is then likely that under the lack of mechanical (masticatory) developmental constraints, nutrition, developmental timing and of course, genetics, have an important effect on mandible shape variation. The effect of nutrition and basal metabolism offers an interesting perspective on malocclusion aetiology, prevention and treatment, and should be looked in deeply. These results also suggest that the relative importance of factors affecting mandible morphology can differ between past and current populations. Consequently, although masticatory loads have been proposed as an important factor determining secular changes among populations sharing the same geographical location [7, 8], the lack of functional constraints due to the modern diet may have led to the emergence of new, more relevant factors that should be taken into consideration.

It is interesting that the amount of cortical bone, on the other hand, seems to behave the opposite than expected. The three low load groups have a larger relative amount of cortical bone. Moreover, their cross section is not particularly elliptical (and thus less able to withstand the torsional loads derived from high masticatory loads). Thus, despite their external gracility, modern mandibles are not

Table 9 Mann-Whitney $U$ pairwise comparison of $\mathrm{I}_{\max }-\mathrm{I}_{\min }$ residuals of the right first molar area

\begin{tabular}{llllll}
\hline & Intense & Medium & Low & Low-class II & Low-class III \\
\hline Intense & & 268 & 160 & 100 & 73 \\
Medium & 0.018 & & 214 & 140 & 103 \\
Low & 0.020 & 0.399 & & 115 & 62 \\
Low-class II & 0.012 & 0.245 & 0.952 & & 49 \\
Low-class III & 0.096 & 0.746 & 0.598 & 0.856 & \\
\hline
\end{tabular}

The upper diagonal shows the values of the $U$ statistic and the lower diagonal the raw $p$ values. After Bonferroni correction, no $p$ values remained significant intrinsically weak. These results could be due to sampling, as statistics do not show striking differences (Tables 4, 6 and 8 ) and the number of individuals with altered occlusion is not large. However, it is worth considering an alternative explanation. Bone structure is dynamic, with cortical and trabecular bone changing in response to load in relatively short periods of time through lifespan [28-30]. In comparison, external morphology changes slowly and, as explained, under the effect of several factors. It is plausible then that with an overall gracile morphology (although not necessarily size), bone seeks to increase its resistance by increasing the amount of cortical bone. Future work could consider changes in trabecular bone structure (trabecular bone thickness, bone volume fraction, orientation, etc.) in order to understand the extent of the effect of masticatory loads on bone morphology.

From a clinical perspective, our study highlights the importance of considering different factors in the aetiology and perhaps treatment of dental malocclusions in relation to an altered mandible shape, particularly since masticatory loads are not always easy to modify. Beyond the more obvious links of our results to orthodontics and surgery, the results regarding the relative amount of cortical bone could be something to take a further look. Mandible crosssection morphology is key in implantology [31], and since there might be a tendency to compensate the gracile mandible shape by increasing the amount of cortical bone, it could be perhaps a good strategy to promote keeping masticatory loads after tooth loss (whenever possible) in order to avoid cortical bone reduction.

In conclusion, the morphology of the human mandible shows a wide range of variation. Its features do not fully support the idea that it is small and weak, leading to malocclusions. We encourage dental practitioners and academics to not take this idea for granted, but to analyse the scenario for each case. Although class II mandibles are indeed smaller, their internal morphology seems to be able to withstand the forces normally produced during chewing. The large variation in the mandible shape of modern individuals could be related to the effect of other factors that, under comparatively low masticatory loads, gain relevance, such as genetics and the largely overlooked effects of nutrition and basal metabolism. 
Acknowledgments The authors would like to thank Jean-Jacques Hublin and Kornelius Kupczik (Max Planck Institute for Evolutionary Anthropology), Iván Pérez (Museo de La Plata), Luis and Cristóbal Quevedo (ICOR), Jacopo Moggi-Cecchi and Monica Zavattaro (Università di Firenze), Markus Bastir (Museo Nacional de Historia Natural de Madrid), Germán Manríquez and Miriam Pérez (Universidad de Chile), Antigoni Kaliontzopoulou (University of Porto), Thomas Püschel (University of Manchester), Adam Sylvester (John Hopkins School of Medicine), Susan Antón and Timothy Bromage (New York University).

Funding Information Open access funding provided by Max Planck Society. The work was funded by Comisión Nacional de Investigación Científica y Tecnológica (Fondo Nacional de Desarrollo Científico y Tecnológico Grant No. 11150175), Chile.

\section{Compliance with ethical standards}

Conflict of interest The authors declare that they have no conflict of interest.

Ethical approval All procedures performed in studies involving human participants were in accordance with the ethical standards of the institutional and national research committee (Comisión Nacional de Investigación Científica y Tecnológica de Chile and the Ethical Committee of Facultad de Medicina Universidad de Chile) and with the 1964 Helsinki declaration and its later amendments or comparable ethical standards.

Informed consent For this type of study, formal consent is not required. Data was provided by the institutions as anonymized files and the methodology was accepted by the relevant ethical committee.

Open Access This article is distributed under the terms of the Creative Commons Attribution 4.0 International License (http:// creativecommons.org/licenses/by/4.0/), which permits unrestricted use, distribution, and reproduction in any medium, provided you give appropriate credit to the original author(s) and the source, provide a link to the Creative Commons license, and indicate if changes were made.

\section{References}

1. Corruccini RS (1984) An epidemiologic transition in dental occlusion in world populations. Am J Orthod 86:419-426. https://doi. org/10.1016/S0002-9416(84)90035-6

2. Andrik P (1963) Die Entwicklung der Bißanomalien vom Neolithikum bis zur Gegenwart. Fortschr Kieferorthop 24:12-21. https://doi.org/10.1007/BF02257681

3. Marangoni A, Belli ML, Caramelli D, Moggi-Cecchi J, Zavattaro M, Manzi G (2011) Tierra del Fuego, its ancient inhabitants, and the collections of skeletal remains in the Museums of Anthropology of Florence and Rome. Museologia Scientifica 5: 88-96

4. El-Zaatari S (2010) Occlusal microwear texture analysis and the diets of historical/prehistoric hunter-gatherers. Int J Osteoarchaeol 20:67-87. https://doi.org/10.1002/oa.1027

5. Larsen CS (2006) The agricultural revolution as environmental catastrophe: implications for health and lifestyle in the Holocene. Quatern Int 150:12-20. https://doi.org/10.1016/j.quaint.2006.01. 004

6. von Cramon-Taubadel N (2011) Global human mandibular variation reflects differences in agricultural and hunter-gatherer subsistence strategies. PNAS 108:19546-19551. https://doi.org/ 10.1073/pnas.1113050108

7. May H, Sella-Tunis T, Pokhojaev A, Peled N, Sarig R (2018) Changes in mandible characteristics during the terminal Pleistocene to Holocene Levant and their association with dietary habits. J Archaeol Sci Rep. https://doi.org/10.1016/j.jasrep.2018. 03.020

8. Galland M, Van Gerven DP, Von Cramon-Taubadel N, Pinhasi R (2016) 11,000 years of craniofacial and mandibular variation in Lower Nubia. Sci Rep 6:31040

9. Pinhasi R, Eshed V, von Cramon-Taubadel N (2015) Incongruity between affinity patterns based on mandibular and lower dental dimensions following the transition to agriculture in the Near East, Anatolia and Europe. PLoS One 10:e0117301. https://doi. org/10.1371/journal.pone.0117301

10. Howe RP, McNamara JA Jr, O'Connor KA (1983) An examination of dental crowding and its relationship to tooth size and arch dimension. Am J Orthod 83:363-373. https://doi.org/10.1016/00029416(83)90320-2

11. Toro-Ibacache V, Cortés Araya J, Díaz Muñoz A, Manríquez Soto G (2014) Morphologic variability of nonsyndromic operated patients affected by cleft lip and palate: a geometric morphometric study. Am J Orthod Dentofac Orthop 146:346-354. https://doi. org/10.1016/j.ajodo.2014.06.002

12. Spassov A, Toro-Ibacache V, Krautwald M, Brinkmeier H, Kupczik K (2017) Congenital muscle dystrophy and diet consistency affect mouse skull shape differently. J Anat 231:736-748. https://doi.org/ 10.1111/joa. 12664

13. Balanta-Melo J, Toro-Ibacache V, Torres-Quintana MA, Kupczik $\mathrm{K}$, Vega C, Morales C, Hernández-Moya N, Arias-Calderón M, Beato C, Buvinic S (2018) Early molecular response and microanatomical changes in the masseter muscle and mandibular head after botulinum toxin intervention in adult mice. Ann Anat 216:112-119. https://doi.org/10.1016/j.aanat.2017.11.009

14. Toro-Ibacache V, Zapata Muñoz V, O'Higgins P (2016) The relationship between skull morphology, masticatory muscle force and cranial skeletal deformation during biting. Ann Anat 203:59-68. https://doi.org/10.1016/j.aanat.2015.03.002

15. Sella-Tunis T, Pokhojaev A, Sarig R, O’Higgins P, May H (2018) Human mandibular shape is associated with masticatory muscle force. Sci Rep 8:6042. https://doi.org/10.1038/s41598-018-242933

16. Bishara SE (2006) Class II malocclusions: diagnostic and clinical considerations with and without treatment. Semin Orthod 12:1124. https://doi.org/10.1053/j.sodo.2005.10.005

17. Joshi N, Hamdan AM, Fakhouri WD (2014) Skeletal malocclusion: a developmental disorder with a life-long morbidity. J Clin Med Res 6:399-408. https://doi.org/10.14740/jocmr1905w

18. Hong SX, Yi CK (2001) A classification and characterization of skeletal class III on etio-pathogenic basis. Int J Oral Maxillofac Surg 30:264-271. https://doi.org/10.1054/ijom.2001.0088

19. Park W, Kim B-C, Yu H-S, Yi C-K, Lee S-H (2010) Architectural characteristics of the normal and deformity mandible revealed by three-dimensional functional unit analysis. Clin Oral Invest 14: 691-698. https://doi.org/10.1007/s00784-009-0349-2

20. Creekmore TD (1967) Inhibition or stimulation of the vertical growth of the facial complex, its significance to treatment. Angle Orthod 37:285-297. https://doi.org/10.1043/0003-3219(1967) 037<0285:iosotv>2.0.co;2

21. Díaz Muñoz A, Manríquez Soto G (2014) Skeletodental diagnosis using a geometric morphometric approach. Int J Odontostomatol 8: 05-11. https://doi.org/10.4067/S0718-381X2014000100001

22. van Spronsen PH (2010) Long-face craniofacial morphology: cause or effect of weak masticatory musculature? Semin Orthod 16:99 117. https://doi.org/10.1053/j.sodo.2010.02.001 
23. Korioth T, Hannam A (1994) Deformation of the human mandible during simulated tooth clenching. J Dent Res 73:56-66. https://doi. org $/ 10.1177 / 00220345940730010801$

24. van Eijden TM (2000) Biomechanics of the mandible. Crit Rev Oral Biol Med 11:123-136. https://doi.org/10.1177/ 10454411000110010101

25. Antón SC, Carter-Menn H, DeLeon VB (2011) Modern human origins: continuity, replacement, and masticatory robusticity in Australasia. J Hum Evol 60:70-82. https://doi.org/10.1016/j. jhevol.2010.08.004

26. Steckel RH (1987) Growth depression and recovery: the remarkable case of American slaves. Ann Hum Biol 14:111-132. https:// doi.org/10.1080/03014468700006852

27. Perez SI, Lema V, Diniz-Filho José Alexandre F, Bernal V, Gonzalez Paula N, Gobbo D, Pucciarelli Héctor M (2011) The role of diet and temperature in shaping cranial diversification of South American human populations: an approach based on spatial regression and divergence rate tests. J Biogeogr 38:148-163. https://doi. org/10.1111/j.1365-2699.2010.02392.x

28. Ruff C, Holt B, Trinkaus E (2006) Who's afraid of the big bad Wolff?:"Wolff's law" and bone functional adaptation. Am J Phys Anthropol 129:484-498. https://doi.org/10.1002/ajpa.20371

29. Barak MM, Lieberman DE, Hublin J-J (2011) A Wolff in sheep's clothing: trabecular bone adaptation in response to changes in joint loading orientation. Bone 49:1141-1151. https://doi.org/10.1016/j. bone.2011.08.020

30. Huang H, Richards M, Bedair T, Fields HW, Palomo JM, Johnston WM, Kim D-G (2013) Effects of orthodontic treatment on human alveolar bone density distribution. Clin Oral Invest 17:2033-2040. https://doi.org/10.1007/s00784-012-0906-y

31. Eufinger H, König S, Eufinger A (1998) The role of alveolar ridge width in dental implantology. Clin Oral Invest 1:169-177. https:// doi.org/10.1007/s007840050029

32. Holmes MA, Ruff CB (2011) Dietary effects on development of the human mandibular corpus. Am J Phys Anthropol 145:615-628. https://doi.org/10.1002/ajpa.21554

33. Zink KD, Lieberman DE, Lucas PW (2014) Food material properties and early hominin processing techniques. J Hum Evol 77:155166. https://doi.org/10.1016/j.jhevol.2014.06.012

34. Morales N, Toro-Ibacache V (2018) The transition to agriculture and industrialization changed the human face. Can vegetarianism be a new factor of change? Review of the literature. Int J Morphol 36:35-40

35. Agrawal KR, Lucas PW, Bruce IC, Prinz JF (1998) Food properties that influence neuromuscular activity during human mastication. J Dent Res 77:1931-1938. https://doi.org/10.1177/ 00220345980770111101

36. Foster KD, Woda A, Peyron MA (2006) Effect of texture of plastic and elastic model foods on the parameters of mastication. $\mathrm{J}$ Neurophysiol 95:3469-3479. https://doi.org/10.1152/jn.01003. 2005

37. Buikstra JE and Ubelaker DH (1994) Standards for data collection from human skeletal remains: Proceedings of a seminar at the Field Museum of Natural History Arkansas Archeological Survey Research Series, Fayetteville

38. Mitteroecker P, Gunz P (2009) Advances in geometric morphometrics. Evol Biol 36:235-247. https://doi.org/10.1007/s11692-0099055-x

39. Toro Ibacache MV, Manriquez Soto G, Suazo Galdames I (2010) Morfometría geométrica y el estudio de las formas biológicas: de la morfología descriptiva a la morfología cuantitativa. Int J Morphol 28:977-990. https://doi.org/10.4067/S0717-95022010000400001

40. Alarcón JA, Bastir M, García-Espona I, Menéndez-Núñez M, Rosas A (2014) Morphological integration of mandible and cranium: orthodontic implications. Arch Oral Biol 59:22-29. https://doi.org/10.1016/j.archoralbio.2013.10.005

41. Halazonetis DJ (2004) Morphometrics for cephalometric diagnosis. Am J Orthod Dentofac Orthop 125:571-581. https://doi.org/10. 1016/S0889540604000319

42. Adams DC, Otárola-Castillo E (2013) geomorph: an r package for the collection and analysis of geometric morphometric shape data. Methods Ecol Evol 4:393-399. https://doi.org/10.1111/2041-210X. 12035

43. Coquerelle M, Bookstein FL, Braga J, Halazonetis DJ, Weber GW, Mitteroecker P (2011) Sexual dimorphism of the human mandible and its association with dental development. Am J Phys Anthropol 145:192-202. https://doi.org/10.1002/ajpa.21485

44. Reno PL, Meindl RS, McCollum MA, Lovejoy CO (2003) Sexual dimorphism in Australopithecus afarensis was similar to that of modern humans. PNAS 100:9404-9409

45. Suazo Galdames IC, Zavando Matamala DA, Luiz Smith R (2008) Evaluating accuracy and precision in morphologic traits for sexual dimorphism in malnutrition human skull: a comparative study. Int $\mathrm{J}$ Morphol 26:876-883

46. Hammer Ø, Harper D and Ryan P (2001) PAST: paleontological statistics software package for education and data analysis. Palaeontol Electron 4:9. doi: Not available

47. Anderson MJ (2001) A new method for non-parametric multivariate analysis of variance. Austral Ecol 26:32-46. https://doi.org/10. 1111/j.1442-9993.2001.01070.pp.x

48. Ito T, Nishimura TD, Hamada Y, Takai M (2015) Contribution of the maxillary sinus to the modularity and variability of nasal cavity shape in Japanese macaques. Primates 56:11-19. https://doi.org/10. 1007/s10329-014-0440-4

49. Klingenberg CP (2011) MorphoJ: an integrated software package for geometric morphometrics. Mol Ecol Resour 11:353-357. https://doi.org/10.1111/j.1755-0998.2010.02924.x

50. Daegling DJ, Grine FE (1991) Compact bone distribution and biomechanics of early hominid mandibles. Am J Phys Anthropol 86: 321-339. https://doi.org/10.1002/ajpa.1330860302

51. Daegling DJ (1993) The relationship of in vivo bone strain to mandibular corpus morphology in Macaca fascicularis. J Hum Evol 25: 247-269. https://doi.org/10.1006/jhev.1993.1048

52. Schneider CA, Rasband WS, Eliceiri KW (2012) NIH Image to ImageJ: 25 years of image analysis. Nat Meth 9:671-675. https:// doi.org/10.1038/nmeth.2089

53. Sokal R, Rohlf F (1995) Biometry. WH Freeman and Company, New York

54. Rothhammer F, Lasserre E, Blanco R, Covarrubias E and Dixon M (1968) Microevolution in human Chilean populations: IV. Shovel shape, mesial-palatal version and other dental traits in Pewenche Indians. Zeitschrift für Morphologie und Anthropologie:162-169. doi: Not available

55. Chrcanovic BR, Abreu MHNG, Custódio ALN (2011) Morphological variation in dentate and edentulous human mandibles. Surg Radiol Anat 33:203-213. https://doi.org/10.1007/ s00276-010-0731-4

56. Katz DC, Grote MN, Weaver TD (2017) Changes in human skull morphology across the agricultural transition are consistent with softer diets in preindustrial farming groups. PNAS 114:90509055. https://doi.org/10.1073/pnas.1702586114

57. Helm JW, German RZ (1996) The epigenetic impact of weaning on craniofacial morphology during growth. J Exp Zool 276:243-253. https://doi.org/10.1002/(SICI)1097-010X(19961101)276:4<243:: AID-JEZ1>3.0.CO;2-O

Publisher's note Springer Nature remains neutral with regard to jurisdictional claims in published maps and institutional affiliations. 